ACTUARIAL RESEARCH CLEARING HOUSE 1980 VOL. 1

\title{
IMMUNIZATION UNDER STOCHASTIC MODELS OF THE TERM STRUCTURE*
}

\author{
By P. P. Boyle, Ph.D., F.I.A., F.C.I.A.
}

\section{INTRODUCTION}

1.1. The purpose of this paper is to survey some new results concerning the term structure of interest rates and discuss actuarial applications. The term structure of interest rates exhibits the relationship among the yields on default free debt instruments of different maturities. Although there is a considerable volume of literature dealing with the determinants of the term structure the analysis has until recently been presented for the most part in a deterministic (i.e. non-stochastic) framework. This constrasts with the treatment of capital assets where equilibrium prices have been obtained under the assumption that returns on these assets are random variables.

1.2. This new approach to the term structure has been developed independently by a number of authors. These include Vasicek, (1) Cox, Ingernoll and Ross, ${ }^{(2)}$ Brennan and Schwartz ${ }^{(3)}$ and Richard. ${ }^{(4)}$ Although the details differ the central idea is to assume that the spot rate $r(f)$ follows a Gaussian Markov process. The arbitrage principle together with some assumption about investors tastes is invoked to obtain the price of a pure discount bond (i.e. a zero coupon bond) of arbitrary maturity. The process followed by the spot rate determines the behaviour of the yield curve. Without empirical testing of these models it is not possible to state which best describes the real world situation.

1.3. There are a number of possible actuarial applications of these results. The present paper examines the concept of immunization within the framework of these models. It will be shown that Redington's(3) basic idea is still valid although there are important differences within the framework of the stochastic model of the term structure. Recently Shedden's' has exiended some of Redington's results to cope with a non-kevel but deterministic yield curve. In the finance literature Fisher and Weil') and Bierwag(s) have studied immunization in the case of coupon paying bonds. Cox, Ingersoll and Roso(") have independently extended the concept of immunization to the case of stochastic models in a manner similar to that developed in $\$ 3$.

\section{STOCHASTIC TERM STRUCTURE MODELS}

2.1. The development given in the first part of this section is a summary of that given by Vasicek. (1) Vasicek's basic assumptions are:

(a) The spot rate follows a continuous Markov process. 
(b) The price $P(t, s)$ at time $t$ of a pure discount bond which matures at time $s$ $(t \leqslant s)$ is determined by the assessment at time $t$, of the segment in $T$. $1<T<s\}$ of the spot rate process over the term of the bond.

(c) The market is efficient and investors act rationally.

2.2. Assumption (a) implies that the spot interest rate $\mathrm{N}(\mathrm{l})$ can be written in the form of a stochastic differential equation

$$
d r=f(r, t) d t+\rho(r, t) d z
$$

where $d z$ is a $W$ iener process with zero mean and variance $d t$. The functions $h(r, t)$ and $[\rho(r, t)]^{2}$ are the instantaneous drift and variance of the process.

2.3. Assumption (b) implies that the bond price is a function of $t, s$ and $r(t)$. Since $(r)$ is a stochastic variable we need to use Ito's lemma ${ }^{(10)}$ to differentiate $P$. Hence

$$
d P=\frac{\partial P}{\partial r} d r+\frac{\partial P}{\partial t} d t+\frac{\partial^{2} P}{\partial r^{2}}[d r]^{2}
$$

2.4. Now we can substitute for $d r$ from (1) in the first term of the right-hand side. Since $d \bar{z}$ is of order $\sqrt{d t}$. $[d r]^{2}=\rho^{2} d t+$ higher order terms in $d t$. Thus [2] becomes

$$
\begin{gathered}
\begin{aligned}
d P=\left[\frac{\partial P}{\partial t}+\right. & \left.f \frac{\partial P}{\partial r}+\frac{1}{2} \rho^{2} \frac{\partial^{2} P}{\partial r^{2}}\right] d t+\rho \frac{\partial P}{\partial r} d z \\
& =P \mu d t+P \sigma d z
\end{aligned} \\
\text { where } \mu=\frac{1}{P}\left[\frac{\partial}{\partial t}+f \frac{\partial}{\partial r}+\frac{1}{2} \rho^{2} \frac{\partial^{2}}{\partial r^{2}}\right] P \\
\text { and } \sigma=\frac{1}{P} \rho \frac{\partial P}{\partial r}
\end{gathered}
$$

Equation (3) holds for bonds of all maturities.

2.5. The development is completed by invoking assumption (c) to prevent riskless arbitrage by reasoning similar to that used to derive the Black Scholes option pricing formula. (1) By constructing a portfolio consisting of two bonds of different maturities Vasicek shows that the ratio $(\mu(t, s)-r(t)) / \sigma(t, s)$ cannot depend on $s$. In particular if this ratio is zero the expected instantaneous returns on all bonds are the same. This corresponds to one version of the Expectations Hypothesis. We shall make this assumption throughout the rest of the paper although it is possible to obtain more general results by assuming a non-zero value for this ratio. Non-zero values can be chosen to correspond to a liquidity premium theory of the term structure.

2.6. The Expectations Hypothesis under these conditions leads to a partial differential equation for the price of a unit discount bond. 
Since $\mu(t, s)=r(t)$ we obtain from equation (4)

$$
\frac{\partial P}{\partial t}+f \frac{\partial P}{\partial r}+\frac{t}{2} \rho^{2} \frac{\partial^{2} P}{\partial r^{2}}-r P=0
$$

2.7. At maturity the bond price is unity by assumption so that $P(s, s, r)=1$ and this furnishes the boundary condition necessary to solve (6). To proceed further it is necessary to make more specific assumptions about the stochastic process (1) followed by the interest rate. Vasicek has suggested the following process

$$
d r=\alpha(\gamma-r) d t+\rho d z
$$

where $\alpha, \gamma$ and $\rho$ are positive constants. This corresponds to the Ornstein-Uhienbeck process and has been used earlier by Beckman ${ }^{(12)}$ to model investment returns. In the present context it has the attractive property that the local interest rate wanders randomly around a long term trend. The further it is away from the trend the stronger is the force which tends to pull it back. Furthermore the mathematics are relatively simple. One disadvantage is that there is a possibility of negative interest rates. However, if the rate does become negative it will be pulled back towards the long-term rate which is positive. To exclude negative interest rates $r=0$ could be made a reflecting barrier but this would probably be somewhat less tractable.

2.8. When the interest rate is given by (7) the solution to (6) obtained by Vasicek is

$$
P(t, s, r)=\exp \left[F(\alpha, T)(D-r)-T D-\frac{\rho^{2}}{4 \alpha}(F(\alpha, T))^{2}\right]
$$

where $T=(s-t)$

$$
\begin{aligned}
F(\alpha, T) & =-\frac{1}{\alpha}(1-\exp (-\alpha \Gamma)) \\
D & =\gamma-\frac{1}{2} \frac{\rho^{2}}{\alpha^{2}}
\end{aligned}
$$

By the properties of the Ornstein-Uhlenbeck process the spot interest rate is normally distributed with conditional mean

$$
E_{n}(r(s))=\gamma+(r(r)-\gamma) \exp (-\alpha T)
$$

and variance

$$
\operatorname{var}(r(s))=\frac{\rho^{2}}{2 \alpha}[1-\exp (-2 \alpha T)]
$$

2.9. Cox, Ingersoll and Ross(2) exclude the possibility of negative interest rates by assuming that the dynamics of the spot rate are given by

$$
d r=x(\mu-r) d t+\sqrt{\sigma^{2} r} d \tilde{z}
$$




\section{Immunizanon Under Siochastic Models of the Term Siruciure}

where $\kappa, \mu$ and $\sigma$ are positive constants. The transition probability density of this process has been derived by Feller ${ }^{\prime 3}$ ' in terms of Bessel functions. The boundary classification criteria have also been developed by Feller. If $2 \kappa \mu / \sigma^{2} \geq l$ the origin is an inaccessible boundary so that negative interest rates are ruled out. Jackson ${ }^{(14)}$ gives a lucid analysis of a number of stochastic models similar to equation (11). The solution to the differential equation (6) obtained by $C$ ox. Ingersoll and Ross $^{(2)}$ for the interest rate dynamics given by $(11)$ is

$$
P(r, I, s):=A(T) \exp (-r B(T))
$$

where

$$
\begin{aligned}
& A(T)=\left[\frac{2 \lambda \exp [(\kappa-\lambda) T] / 2}{(\lambda+\kappa)(1-\exp (-\lambda T)+2 \lambda \exp (-\lambda T)}\right]^{\frac{2 \kappa \mu}{\sigma^{2}}} \\
& B(T)=\frac{2(1-\exp (-\lambda T)}{\{(\lambda+\kappa)[1-\exp (-\lambda T)]+2 \lambda \exp (-\lambda T)\}} \\
& \lambda^{2}=\kappa^{2}+2 \sigma^{2}
\end{aligned}
$$

2.10. Both of the solutions (8) and (12) give yield curves which are increasing for low values of the current rate $r(t)$ and decreasing for high values of the current rate. In each case there is a range of values which produces a humped yield curve.

2.11. Readers unfamiliar with the methods mentioned in this section will find helpful background material in Cox and Millar's' and a more advanced approach in McKean. ${ }^{(10)}$

\section{MATCHING OF ASSETS AND LIABILITIES-IMMUNIZATION}

3.1. The importance of the maturity structure of the liabilities in selecting appropriate assets has been widely recognized in actuarial circles. The classic paper is by Redington ${ }^{(5)}$ who showed that under certain assumptions it was possible to develop an immunization strategy. It will be shown in this section that the thrust of Redington's approach is still valid under the equilibrium theories of the term structure just outlined. The actual term structure model used to derive Redington's result does not represent an equilibrium situation since it admits arbitrage profits. This point was alluded to by $C$. D. Rich in the discussion of Redington's paper. It is hoped that the analysis presented here will resolve this paradox.

3.2. First we restate Redington's basic result. Assume that an insurance company's liabilities can be represented by

$$
L_{T} \quad(O \leqslant T \leqslant \infty)
$$

where $L_{\mathrm{r}}$ represents the net liability payment at time $T$ hence. It is assumed that $L_{r}$ is known with certainty. In the same way $A_{r}$ denotes the asset proceeds 
payable at time $T$. Let $\delta$ (assumed constant) represent the force of interest (i.e. the interest rate under continuous compounding, following conventional actuarial notation). Assume that the yield curve is level. Suppose

$$
V A=V L
$$

where $V A=\int_{0}^{\infty} A T \exp (-\delta T) d T$

and $V L=\int_{0}^{\infty} L_{T} \exp (-\delta T) d T$

If in the next instant $\delta$ changes by a small amount $\varepsilon$ the new values of $V A$ and $V L$ are $V A^{\prime}$ and $V L^{\prime}$. By using a Taylor series expansion for $V A^{\prime}-V L^{\prime}$, Redington showed that as long as

$$
\frac{\partial V A}{\partial \delta}=\frac{\partial V L}{\partial \delta}
$$

and

$$
\frac{\partial^{2} V A}{\partial \delta^{2}}>\frac{\partial^{2} V L}{\partial \delta^{2}}
$$

then

$$
V A^{\prime}>V L^{\prime}
$$

3.3. As a simple illustration, consider a single premium pure endowment due in 10 years. Ignore mortality. The face value of the contract is 100 and the liability is met by investing part of the premium in a S-year discount bond and the balance in a 15 -year discount bond. If $\delta=0.08$ then the value of the endowment is

$$
100 \exp (-.8)=44.9328964
$$

To achieve immunization the amounts of the premium to be invested in the 2 bonds are given by the solution of the following pair of simultaneous equations. (The 5-year bond promises $\lambda_{1}$ units at maturity while the 15-year bond promises $\lambda_{2}$ units at maturity.)

$$
\begin{gathered}
\lambda_{1} \exp (-.4)+\lambda_{2} \exp (-1 \cdot 2)=100 \exp (-.8) \\
5 \lambda_{1} \exp (-.4)+15 \lambda_{2} \exp (-1 \cdot 2)=1000 \exp (-.8)
\end{gathered}
$$

This gives

$$
\begin{aligned}
& \lambda_{1}=50 \exp (-.4)=33.5160023 \\
& \lambda_{2}=50 \exp (.4)=74.5912349 .
\end{aligned}
$$


In this case the value of $\frac{\partial^{2} V A}{\partial \delta^{2}}-\frac{\partial^{2} V L}{\partial \delta^{2}}$ is

$$
\begin{gathered}
25 \lambda_{1} \exp (-4)+225 \lambda_{2} \exp (-1 \cdot 2)-10000 \exp (-8) \\
=1250 \exp (-8)[1+9-8]>0
\end{gathered}
$$

3.4. In the absence of transactions costs this result implies the existence of arbitrage profits for an investor who can borrow and lend at the same rate. The underlying model of the term structure is not an equilibrium model. It is of some interest therefore to approach the immunization problem within the framework of the term structure models developed in $\$ 2$.

3.5. First, however, some brief comments are in order. Notice that the arbitrage situation given in the example does not depend on the particular numerical values selected. A more general algebra ic development is possible. Also note that the statement $\delta$ changes to $(\delta+\varepsilon)$ in the next instant was somewhat loose. This really means that $\delta$ has stochastic properties and that if $\delta$ is a continuous function of time a change in $\delta$ may induce additional changes in the value of discount bonds because time to maturity has been reduced.

3.6. Recall from $\$ 2$ that under uncertainty a pure discount bond of maturity $T$ has value $P(r, T)$ where $r$ is the current spot interest rate and $P$ satisfies equation (6). If assets and liabilities are perfectly matched

$$
\begin{gathered}
A T=L_{T} \text { for all } T \\
\text { then } \int_{0}^{\infty} P\left(r, \lambda_{1} A_{T} d T=\int_{0}^{\infty} P(r, T) L_{T} d T\right.
\end{gathered}
$$

In this case of absolute matching the fund is perfectly immunized irrespective of the bond pricing process.

3.7. It is still possible to achieve an immunized position when bond prices are given by $P(r, T)$ without going to the extreme of absolute matching. What is not possible, however, is to obtain arbitrage profits in these circumstances. This is not surprising since arbitrage arguments played a decisive role in setting up the equation for the bond price in the first place. The immunized position conditional on the current rate $r$ is obtained by arranging that the stochastic component vanishes. If

$$
V A=V L
$$

where $V A=\int P(r, T) A_{I} d T$

$$
V L=\int P(r, T) L_{T} d T \text {. }
$$

the stochastic component of the return will be [from (3)]

$$
\left(\rho \frac{\partial V A}{\partial r}-\rho \frac{\partial V L}{\partial r}\right) d z
$$


By selecting the assets so that

$$
\frac{\partial V A}{\partial r}=\frac{\partial V L}{\partial r}
$$

the position will be immunized and the total return will be zero.

3.8. Notice that if (15) alone is satisfied the presence of the non-zero stochastic component indicates that the expected return will zero $(E(d z)=0)$. Further the immunization position given by (17) is valid only for the current interest rate and the current maturity structure of the liabilities. The immunization position has to be adjusted continuously to accommodate changes in these parameters.

Notice also that to obtain

$$
\frac{\partial V L}{\partial r}
$$

we need to know

$$
\frac{\partial P}{\partial r}(r, T)
$$

for all maturities $T$ of interest. This partial derivative will be obtained from an equation like equation (8) (Vasicek) ${ }^{11}$ or equation (12) (Cox, Ingersoll and Ross). ${ }^{(2)}$

The mean term of the assets is given by

$$
-\frac{\frac{\partial V A}{\partial r}}{V A}
$$

In the finance literature this concept is known as duration. To achieve immunization under the stochastic models the mean term of the assets has to be made equal to the mean term of the liabilities as in Redington's theory.

3.9. To illustrate numerically some of the differences between the term structure models considered, Table 1 has been prepared. This table gives the prices of pure discount bonds ( $100=$ maturity value) for maturities of 5,10 and 15 years. The mean terms of the bonds are also given. The parameter values for the Vasicek model and the Cox, Ingersoll and Ross model have been deliberately selected to provide broad agreement between these two models. To achieve this the longterm mean in both cases has been set equal to 07 . The speed of adjustment is the same in both cases, $\alpha=\kappa=1$ and when $r=.07$ the infinitesimal variances are equal

$$
\sigma^{2} \times \cdot 07=\cdot 0002=\rho^{2}
$$


Table 1. Bond Prices and Mean Terms of Discount Bonds (Face Value $=100)$ ) under Various Term Structure Models

(i) Flat Yield Curve

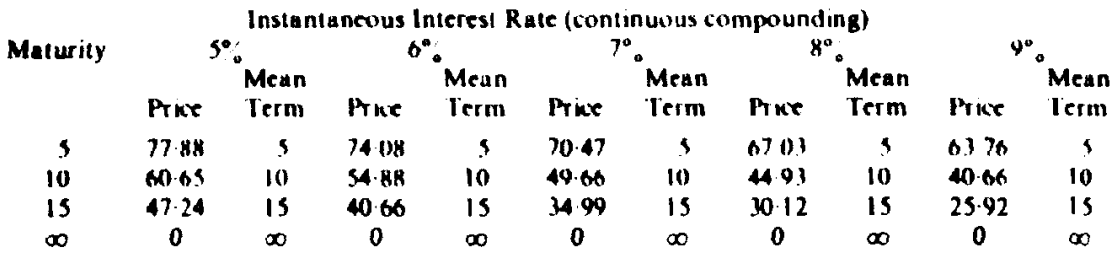

(ii) Vasicek Model $\rho^{2}=0002, \gamma=07, \alpha=\cdot 1$

\begin{tabular}{cccccccccccc} 
Maturity & \multicolumn{2}{c}{$5 \%$} & \multicolumn{2}{c}{$6 \%$} & \multicolumn{2}{c}{$7 \%$} & \multicolumn{2}{c}{$8 \%$} & \multicolumn{2}{c}{$9 \%$} \\
5 & 76.46 & 3.93 & 73.51 & 3.93 & 70.67 & 3.93 & 67.95 & 3.93 & 65.33 & 3.93 \\
10 & 57.31 & 6.32 & 53.79 & 6.32 & 50.50 & 6.32 & 47.41 & 6.32 & 44.50 & 6.32 \\
15 & 42.64 & 7.77 & 39.45 & 7.77 & 36.50 & 7.77 & 33.77 & 7.77 & 31.25 & 7.77 \\
$\infty$ & 0 & 10 & 0 & 10 & 0 & 10 & 0 & 10 & 0 & 10
\end{tabular}

(ii) Cox. Inger soll, Ross model $\sigma^{2}=002857, \mu=07, x=\cdot 1$

\begin{tabular}{cccccccccccc} 
Maturity & \multicolumn{2}{c}{$5 \%$} & \multicolumn{2}{c}{$6 \%$} & \multicolumn{2}{c}{$7 \%$} & \multicolumn{2}{c}{$8 \%$} & \multicolumn{2}{c}{$9 \%$} \\
5 & 76.40 & 3.90 & 73.48 & 3.90 & 70.67 & 3.90 & 67.97 & 3.90 & 65.37 & 3.90 \\
10 & 57.07 & 6.14 & 53.67 & 6.14 & 50.47 & 6.14 & 47.46 & 6.14 & 44.64 & 6.14 \\
15 & 42.21 & 7.39 & 39.20 & 7.39 & 36.41 & 7.39 & 33.81 & 7.39 & 31.40 & 7.39 \\
$\infty$ & 0 & 8.87 & 0 & 8.87 & 0 & 8.87 & 0 & 8.87 & 0 & 8.87
\end{tabular}

3.10. Notice that the prices given by models (ii) and (iii) are very similar as we would expect by construction. For current interest rates below the long-term mean the stochastic models give lower bond prices than those given by the deterministic model (i). For high current interest rates the reverse is true. These features result from the mean reversion property of the interest rate in the stochastic models.

3.11. Under the deterministic model the mean term of a $T$ period bond is equal to $T$. In the stochastic models the mean term of a $T$ year bond is a more complicated function of $T$ and in both cases it is bounded as $T \rightarrow \infty$. For model (ii) the limiting value of the mean term for a very long bond is

$$
\operatorname{Lim}_{T \rightarrow \infty} \frac{(1-\exp (-\alpha T))}{\alpha}=\frac{1}{\alpha}
$$

In the case of model (iii) the limit is

$$
\frac{2}{\sqrt{\kappa^{2}+2 \sigma^{2}}+\kappa}
$$

3.12. It may be of interest to obtain the immunized strategy for the 10-year single premium pure endowment policy discussed earlier. The amounts $\lambda_{1}$ and $\lambda_{2}$ 
for a given (current) interest rate $r(t)$ and a specified term structure model are obtained by solving

$$
\begin{aligned}
& \lambda_{1} P(r, 5)+\lambda_{2} P(r, 15)=100 P(r, 10) \\
& \lambda_{1} \frac{\partial P}{\partial r}(r, 5)+\lambda_{2} \frac{\partial P}{\partial r}(r, 15)=10 \frac{\partial P}{\partial r}(r, 10)
\end{aligned}
$$

For the deterministic model $P(r, T)=\exp (-r \eta)$ and for models (ii) and (iii) $P(r, N)$ is given by equations (8) and (12). The values of $\lambda_{1}$ and $\lambda_{2}$ for the parameter values used to obtain Table $l$ are given in Table 2 . While the immunization strategies do not differ too much between the stochastic models, the results for model (i) are quite different from the other two. The stochastic models require greater investment in the 15-year bond.

Table 2: Amounts of 5-year and 15-year Discount Bonds to Immunizea 10-year Single Premium Pure Endowment (mortality ignored). The Face Value of the Endowment is 100

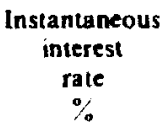

\begin{tabular}{|c|c|}
\hline \multicolumn{2}{|c|}{$\begin{array}{l}\text { Model (i) } \\
\text { deterministic } \\
\text { flat lerm } \\
\text { structure }\end{array}$} \\
\hline $\begin{array}{c}\lambda_{1} \\
38.94\end{array}$ & $\begin{array}{c}\lambda_{2} \\
64 \cdot 20\end{array}$ \\
\hline 37.41 & 67.49 \\
\hline $35 \cdot 23$ & 70.95 \\
\hline 33.52 & \\
\hline 31.88 & 78.4 \\
\hline
\end{tabular}

5
6
7
8
9

\begin{tabular}{|c|c|}
\hline \multicolumn{2}{|c|}{$\begin{array}{l}\text { Model (iii) } \\
\text { Cox, Ingersoll, Ross } \\
\text { parameter values } \\
\text { in Table } 1\end{array}$} \\
\hline $\begin{array}{c}\lambda_{1} \\
26 \cdot 72 \\
26 \cdot 12 \\
25 \cdot 54 \\
24 \cdot 98 \\
24.42\end{array}$ & $\begin{array}{c}\lambda_{2} \\
86.84 \\
87.93 \\
89.04 \\
90 \cdot 14 \\
91 \cdot 30\end{array}$ \\
\hline
\end{tabular}

$\begin{array}{cc}\begin{array}{c}\text { Model (ii) } \\ \text { Vasicel }\end{array} \\ \text { parameter values } \\ \text { in Table i } \\ \lambda_{1} & \lambda_{2} \\ 28.29 & 83.66 \\ 27.63 & 84.88 \\ 26.98 & 86.12 \\ 26.34 & 87.38 \\ 25.72 & 88.65\end{array}$

3.13. To see why the stochastic models give rise to relatively greater investment in the long-term asset the following comments may be helpful. Under the deterministic model a small change in the local interest rate induces a movement in the level of the entire yield curve. Thus it can have a dramatic impact on the price of a long-term bond. In this model the price of a long bond is very sensitive to interest rate changes. The mean term which can be taken as a measure of the riskiness of the bond with respect to movements in the interest rate is equal to $T$, the time to maturity. In both stochastic models the local interest rate displays mean reversion. Thus changes in the spot rate have a more pronounced effoct on the yields of shorter dated bonds. In model (ii) the mean terms of the 5-, 10-and 15-year bonds are 3.93,6.32 and 7.77. The increase in riskiness in moving to longer maturities is not as dramatic as under the deterministic model. When the immunization strategy is implemented it is not surprising to find that the stochastic model gives rise to greater investment in the longer-term bond. 


\section{CONCLUSION}

4.1. The immunization strategy just outlined depends of course on the validity of the underlying model. To implement it correctly one needs also to know the value of the underlying parameters which give the local dynamics of the spot rate Nevertheless it is contended that the stochastic approach provides a valuable approach to the problem.

4.2. It is worth pointing out that by assuming that the net liability outfow is certain at time $T$ we are ignoring mortality and other contingencies. In the case of a company with a large portfolio of contracts this procedure can be justified by an appeal to the law of large numbers. It is usual to think of the law of large numbers as being required to reduce mortality risk. In the present context it is also required (indirectly) to reduce investment risk.

4.3. There are other problems in actuarial science which can be attacked within the framework of a stochastic term structure. These include a range of situations where financial options are granted to an insured. For example an endowment insurance maturing at age 65 may include guaranteed conversion rates (to a life annuity). The valuation of an option of this type could be placed on a more scientific basis within the framework of a stochastic model of interest rates. In fact many of the features and riders found in insurance contracts can be regarded as options. These may involve financial options, mortality options or combinations of the two. It is suggested that the stochastic models discussed in this paper will prove useful in pricing such options.

4.4. I am grateful to my colleagues, Professors J. D. Murray and M. J. Brennan for useful comments.

\section{REFERENCES}

(1) VAsscex, O. A. An Equilibrium Characterization of the Term Structure. Journal of Financial Eramamics, 5 (November 1977).

(2) Cox. J. C.. Inomsoll, J. E. Ross, S. A. A Theory of the Term Structure of Interest Rates and the Valuation of Interest-Dependent Claims. (Paper presented at the Western Finance Associntion Meetings. Anaheim. Calidornic. June 1977).

(3) Brannan, M. J. Scrwwakrz, E. S. Savings Bonds, Retractable Bonds and Callable Bonds. Jowrmol of Finoncial Economics. S. 67-88 (1977).

(4) Ruchard. S. F. An Analytical Model of the Term Structure of Interest Rates. (Working Paper, Graduate School of Industrial Administration, Carnegie-Mellon University, Pittsbur gh, Pennsylvania, December 1976).

(5) Redington, F. M. Review of the Principles of Life Office Valuations. J.I. A., 78, 286.

(6) Shedden, A. D. A Practical Approach to Applying Immunization Theory. T.F.A., 35. 313.

(7) Fishex, L. WeW. R. L. Coping with the Risk of Interest Rate Fluctuations. Joumal of Business 4., 408.

(9) Berwag, G. O. Immunization Duration and the Term Suructure of Interest Rates. Jowrnal of Financial and Quantilative Amalysis (forthcoming).

(9) Cox. J. C.. Inoensolt, J. E. \&oss. S. A. Duration and the Measurement of Basis Risk. (Mimeograph. Graduate School of Business, University of Chicago. Jllinois, July 1977 ).

(10) McKean, H. P.. Jx., Stochastic Iniegrals. Academic Press, New York. 1969. 
Immunization Under Stochastic Models of the Term Structure

(II) Black, F. \& Scholes. M. J. The Pricing of Options and Corporate Liabilities. Journal of Political Economy 81, 637

(12) Beekman. J. A. A new Collective Risk Model. T.S.A. XXV, 573.

(13) Fellen, W. Two Singular Diffusion Problems. Annals of Mathemarics 4 , 173.

(14) Jackson. C. J. Stochastic Models of a Risk Business Operating Under the Influence of Investment and Insurance Risks. (Ph.D. thesis, Graduate School of Business, University of Wisconsin-Madison, (971)

(I5) Cox. D. R. \& Mili.AR. H. D. The Theory of Stochasfic Processes Methuen. London. 1965. 
